\title{
Tratamiento basado en atención plena compasiva: un estudio piloto en policonsumidores de drogas ${ }^{1}$
}

\section{Treatment based on compassionate mindfulness: A pilot study in drugs polyconsumers}

\author{
José Luis Barrera Gutiérrez², Diana Mejía Cruz ${ }^{3}$ \\ y Ana Beatriz Moreno Coutiño ${ }^{2}$
}

\begin{abstract}
Citación: Barrera Gutiérrez, J.L., Mejía C., D. y Moreno Coutiño, A.B. (2021). Tratamiento basado en atención plena compasiva: un estudio piloto en policonsumidores de drogas. Psicología y Salud, 31(1), 25-35. https://doi.org/10.25009/pys.v31i1.2673.
\end{abstract}

\section{RESUMEN}

\begin{abstract}
La Organización Mundial de la Salud refiere que el consumo de drogas psicoactivas es un problema relevante de salud pública en el mundo ya que genera graves consecuencias a nivel individual, familiar y social. En México, de acuerdo con la Encuesta Nacional de Consumo de Drogas, Alcohol y Tabaco, ha habido un incremento en el consumo de drogas legales e ilegales entre los años 2011 y 2016, reportando dependencia a drogas el $0.6 \%$ de la población de 12 a 65 años. Se ha encontrado que la mayoría de los consumos de sustancias están relacionadas a factores emocionales como el estrés, los afectos negativos y la sintomatología depresiva y ansiosa. Como parte de las terapias de tercera generación, la de atención plena ha despertado un creciente interés desde 1990 en el campo científico en cuanto que se relaciona con mejoras en la satisfacción de vida, felicidad, reducción de depresión y ansiedad, entre otras psicopatologías (Pérez, 2006). De igual manera, ha sido llevada al campo de las conductas adictivas, demostrando eficacia y efectividad en el tratamiento del consumo de sustancias. El objetivo de esta investigación fue probar los efectos de un tratamiento psicológico basado en la atención plena compasiva sobre la sintomatología ansiosa y depresiva, la felicidad, la compasión y el consumo de sustancias, para lo cual se utilizó un diseño intrasujetos, administrándose a cuatro pacientes con trastornos por consumo de sustancias en una intervención grupal de nueve sesiones. Se tomaron medidas basales, en el postratamiento y el seguimiento a un mes, calculándose el tamaño del efecto clínico de cada una de esas variables. Los resultados sugieren que la intervención de atención plena compasiva fue eficaz para reducir la sintomatología ansiosa y depresiva, la felicidad fluctuante y el patrón de consumo, e incrementar la felicidad duradera y la compasión.
\end{abstract}

Palabras clave: Atención plena; Compasión; Drogas; Felicidad; Sintomatología ansiosadepresiva.

\begin{abstract}
The World Health Organization points out that the use of psychoactive drugs is a relevant public health problem in the world because it leads serious individual, family, and social consequences. In Mexico, according to the National Survey of Drugs, Alcohol, and Tobacco consumption 2016-2017, there are increases in consumption of legal and illegal drugs between 2011 and 2016. Actual full drug dependence affects $0.6 \%$ of the total population aged 12 to 65 years. Most substance use seems
\end{abstract}

\footnotetext{
${ }^{1}$ Los autores agradecen al Consejo Nacional de Ciencia y Tecnología por el financiamiento al protocolo doctoral del primer autor a través del CVU: 323904, con número de becario 230035 y de beca 449025, del cual se desprende este estudio. Se extiende un agradecimiento especial a la M.C. Silvia Valle por las facilidades otorgadas en la realización de esta investigación en el Centro de Tratamiento Residencial y Ambulatorio, así como a la Psic. Andrea Romero Ovalle por su apoyo en las sesiones de tratamiento.

${ }^{2}$ Facultad de Psicología, Universidad Nacional Autónoma de México, Av. Universidad 3004, Col. Copilco Universidad, Del. Coyoacán, 04510 Ciudad de México, México, correos electrónicos: moca99_99@yahoo.com y psicopumatkd@hotmail.com. Artículo recibido el 15 de octubre de 2019 y aceptado el 30 de mayo de 2020.

${ }^{3}$ Departamento de Psicología, Instituto Tecnológico de Sonora, 5 de febrero 818 Sur, 85000 Cd. Obregón, Son., México, correo electrónico: diana.mejia@itson.edu.mx.
} 
related to such emotional factors, as stress, negative affect, and depressive and anxious symptoms. As a part of third-generation therapies, mindfulness has received growing scientific interest since the 1990s because it is related to the improvements in life satisfaction and happiness, as well as to the reduction of depression and anxiety, among other disorders. Mindfulness has also been applied to the field of addictive behaviors and shown effectiveness at treating substance use. The objective of the present study was to test the effects of a psychological treatment based on compassionate mindfulness on anxious and depressive symptomatology as well as happiness, compassion, and substance use patterns. An within subjects design was used, to evaluate a group intervention of nine sessions to four patients with substance use disorder. Baseline, post-treatment and one month follow-up measures were taken, and the size of the clinical effect was computed for each variable. Results suggest that compassionate mindfulness intervention was effective in reducing anxious and depressive symptoms, fluctuating happiness, and consumption pattern, and increasing longer-lasting happiness and compassion.

Keywords: Mindfulness; Compassion; Drugs; Happiness; Depressive anxious symptomatology.

\section{INTRODUCCIÓN}

\section{Tratamientos para el consumo de sustancias}

E 1 consumo de drogas psicoactivas es un problema relevante de salud pública nacional e internacional; de acuerdo con los datos obtenidos por la United Nations Office for Drugs and Crime (UNODC) (2020), se estima que cerca de 29.5 millones de personas padecen diversos problemas provocados por el uso de sustancias (cf. Secretaría de Salud y Asistencia Instituto Nacional de Psiquiatría Ramón de la Fuente Muñiz, Instituto Nacional de Salud Pública y Comisión Nacional contra las Adicciones, 2017).

En México, según datos de la Encuesta Nacional de Consumo de Drogas, Alcohol y Tabaco (ENCODAT 2016-2017), se reporta que 0.6\% de la población de 12 a 65 años es dependiente de las drogas durante el último año, siendo más grave el problema en los hombres ( $2 \%$ ), que en las mujeres $(0.3 \%)$, en edades que oscilan entre 18 y 34 años (SSA, 2017).

Los programas de tratamiento para el consumo de sustancias incorporan típicamente una multiplicidad de orientaciones, que van desde aproximaciones meramente farmacológicas hasta enfoques psicológicos con orientaciones diversas: tratamientos psicodinámicos, humanistas, sistémicos, cognitivo-conductuales de segunda generación o integrales. Sin embargo, no todos los tratamientos son eficaces (Allen, Litten y Fertig, 1995; Rojas, Real, García y Medina-Mora, 2011).

Autores como Pascual, Frías y Monterde (2004) han mostrado los siguientes criterios que un estudio debe tener para tener mayor eficacia: a) asignación aleatoria de los participantes a las condiciones de tratamiento y control; b) utilización de métodos de ciego y doble ciego; $c$ ) aplicación de las intervenciones por terapeutas capacitados; d) número fijo de sesiones, y e) periodos fijos de tiempo para que los pacientes puedan ser seguidos después del tratamiento. Las intervenciones llevadas a cabo mediante la terapia cognitiva-conductual (TCC) son las que cumplen con la mayoría de los criterios anteriormente mencionados. Desde este enfoque se han desarrollado numerosos programas de tratamiento contra el consumo de sustancias, reportándose resultados positivos (Carroll y Kiluk, 2017; Magill et al., 2019; Pirnial et al., 2019; Witkiewitz, Marlatt y Walker, 2005). En México, los programas que prestan atención al problema de las adicciones están basados, en su mayoría, en intervenciones y tratamientos con la citada orientación (Ayala, Echeverría, Sobell y Sobell, 1988; Barragán, Flores, Morales, González y Martínez, 2006; Barragán, González, Medina y Ayala, 2005; Echeverría, Ruiz, Salazar y Tiburcio, 2008; Martínez, Salazar, Ruiz, Barrientos y Ayala, 2007; Oropeza, Medina-Mora y Sánchez-Sosa, 2007). Sin embargo, entre las limitaciones más importantes de dicho modelo se encuentran la falta de mantenimiento de los resultados a largo plazo y las recaídas en aproximadamente dos terceras partes de los usuarios bajo tratamiento (Gross y Levenson, 1993, 1997; Gutiérrez, Luciano, Rodríguez, y Fink, 2004; Hayes, 2004; Moreno 2012; Rojas et al., 2011; Zgierska et al., 2009).

Debido a lo anterior, resulta necesario continuar desarrollando nuevas y más eficaces aproximaciones para el tratamiento del consumo de sustancias. Actualmente, las terapias basadas en la atención plena (mindfulness) han probado ser muy eficaces en el tratamiento de diversas adicciones (Chiesa y Serretti, 2014; Karyadi, Vander- 
Veen y Cyders, 2014; Schuman-Olivier, Hoeppener, Evins y Brewer, 2014; Witkiewitz, Marlatt y Walker, 2005).

\section{Atención plena como tratamiento del consumo de sustancias}

Dentro de las estrategias mundiales para atender el abuso de sustancias se ha propuesto generar tratamientos basados en evidencia para asegurar que la abstinencia persista, y los concernientes a atención plena han demostrado eficacia y efectividad para tratar el problema (Brewer, Elwafi y Davis, 2013; Chiesa y Serretti, 2014; Karyadi, VanderVeen y Cyders, 2014; Marlatt y Kristeller, 1999; Oikonomou, Arvanitis y Sokolove, 2016; Schellhas, Ostafin, Palfai y De Jong, 2016; Schuman-Olivier, Hoeppener, Evins y Brewer, 2014; Witkiewitz, Marlatt y Walker, 2005; Zgierska et al., 2009).

Las personas que abusan de sustancias experimentan numerosos pensamientos, sentimientos y sensaciones físicas desagradables que precipitan un uso más frecuente o más insistente. El consumo de sustancias y otras conductas adictivas sirven a menudo para evitar o controlar esas experiencias internas, así como la incomodidad asociada a ellas (Bowen, De Boer y Bergman, 2017; Stotts y Northrup, 2015).

Un objetivo general de las terapias de tercera generación (o TTG) es identificar y experimentar formas alternativas de relacionarse con esos eventos privados desagradables, formas que interumpirían el vínculo aparentemente causal entre la angustia psicológica y física y el consumo de drogas. Por ejemplo, las habilidades de aceptación se utilizan para facilitar una mayor voluntad de vivir pese a los acontecimientos angustiosos, y las estrategias de regulación de emociones se pueden emplear para aumentar la conciencia emocional y reducir la vulnerabilidad ante las emociones abrumadoras (Hayes, 2004; Karyadi, VanderVeen y Cyders, 2014; Marlatt y Kristeller, 1999).

Las intervenciones de atención plena aumentan la conciencia de las contingencias ambientales naturales y ayudan a los consumidores de sustancias a advertir los factores desencadenantes y las consecuencias de una manera flexible y no reactiva. Idealmente, en este contexto nuevo y cons- ciente es posible que se desarrollen las conductas necesarias para tomar decisiones y elecciones basadas en metas y valores personales importantes, en lugar de evitar o controlar el consumo de sustancias. En otras palabras, más que centrarse exclusivamente en la reducción y eliminación de conductas adictivas, las TTG ponen énfasis en la vida valiosa y significativa, buscando identificar los valores que posee el consumidor para dignifi- $\square$ car el sufrimiento inevitable que implica el abandono de un comportamiento bien establecido de consumir sustancias, el cual servía como un escape de la angustia y de las consecuencias de la adicción (Stotts y Northrup, 2015).

Dados los antecedentes documentados sobre los beneficios registrados de las intervenciones basadas en la atención plena y su componente compasivo (Simkin y Black, 2014), en el presente estudio se efectuó una intervención piloto basada en la atención plena compasiva para reducir el consumo de sustancias, las sintomatologías ansiosa y depresiva y la felicidad fluctuante, así como para aumentar la felicidad duradera y la compasión en policonsumidores con tratamiento ambulatorio.

\section{MÉTODO}

\section{Diseño}

A este efecto, se utilizó un diseño preexperimental intrasujetos $(\mathrm{N}=4)$, A-B-C prospectivo con línea base, con medición posterior a la intervención y seguimiento a un mes. Tal diseño es especialmente apropiado para los estudios de corte clínico, en los cuales se documentan el sentido y el tamaño del cambio ante la implementación sistemática de una variable independiente.

\section{Participantes}

Participaron en el estudio cuatro pacientes de un centro de atención residencial en modalidad ambulatoria, del sexo masculino, mayores de edad, con trastorno por consumo de moderado a severo de múltiples sustancias, cuyas características sociodemográficas y criterios de inclusión y exclusión se muestran en las Tablas 1 y 2. 
Tabla 1. Características sociodemográficas.

\begin{tabular}{|c|c|c|c|c|c|c|c|}
\hline Caso & Edad & $\begin{array}{c}\text { Estado } \\
\text { civil }\end{array}$ & Ocupación & $\begin{array}{c}\text { Escolaridad } \\
\text { (años) }\end{array}$ & Religión & $\begin{array}{c}\text { Puntaje } \\
\text { CAD 20 }\end{array}$ & $\begin{array}{c}\text { Puntaje } \\
\text { EDA }\end{array}$ \\
\hline 1 & 18 & Soltero & Desempleado & 7 & Católica & 15 & 11 \\
\hline 2 & 22 & Soltero & Estudiante & 14 & Católica & 11 & 3 \\
\hline 3 & 27 & Soltero & Empleado & 9 & Católica & 14 & 3 \\
\hline 4 & 33 & Soltero & Empleado & 14 & Creyente & 11 & 19 \\
\hline
\end{tabular}

Nota: El Cuestionario de Abuso de Drogas (CAD-20) es un autoinforme compuesto por veinte preguntas de respuesta dicotómica. Proporciona un índice cuantitativo del rango de problemas asociados con el abuso de drogas (De las Fuentes y Villalpando, 2001).

La Escala de dependencia al Alcohol (EDA) es un instrumento de 25 reactivos que mide la dependencia de baja a severa (Ayala, Echeverría y Gutiérrez, 1998).

Tabla 2. Criterios de inclusión y exclusión.

\begin{tabular}{|l|l|l|}
\hline \multicolumn{1}{|c|}{ Inclusión } & \multicolumn{1}{|c|}{ Exclusión } & \multicolumn{1}{c|}{ Eliminación } \\
\hline $\begin{array}{l}\text { Sujetos de sexo masculino } \\
\text { de 18 a 65 años de edad. }\end{array}$ & $\begin{array}{l}\text { Padecer algún trastorno psiquiátrico diagnosti- } \\
\text { cado mediante la Entrevista Neuropsiquiátrica } \\
\text { Internacional (MINI) (Ferrando, Bobes, Gibert, } \\
\text { Soto y Soto, 2000). }\end{array}$ & $\begin{array}{l}\text { Deserción una vez iniciado } \\
\text { el estudio. }\end{array}$ \\
\hline $\begin{array}{l}\text { Diagnóstico de dependencia } \\
\text { a múltiples sustancias, determinada } \\
\text { por el psicólogo tratante del servicio } \\
\text { de impresión diagnóstica. }\end{array}$ & $\begin{array}{l}\text { Hallarse en tratamiento farmacológico, médico } \\
\text { o psicológico al momento en que se inició } \\
\text { la intervención y hasta tres meses antes. }\end{array}$ & $\begin{array}{l}\text { Llenado incompleto de los } \\
\text { instrumentos de evaluación. }\end{array}$ \\
\hline $\begin{array}{l}\text { Consumo de sustancias en los últimos } \\
\text { tres meses. }\end{array}$ & Ser practicante de la meditación. \\
\hline $\begin{array}{l}\text { Aceptación a participar en el estudio } \\
\text { y firma de la carta de consentimiento } \\
\text { informado. }\end{array}$ & $\begin{array}{l}\text { Faltar a alguna de las nueve sesiones } \\
\text { de tratamiento. }\end{array}$ & No someterse a prueba antidoping. \\
\hline
\end{tabular}

\section{Instrumentos}

Marcador biológico: Prueba de orina DrugScreenDip Test para detectar cinco drogas (marihuana, cocaína, opiáceos, anfetaminas y metanfetaminas) de AMEDICA ${ }^{\circledR}$ DrugScreen.
Pruebas psicométricas: Las pruebas utilizadas y sus propiedades psicométricas se describen en la Tabla 3.

Tabla 3. Características psicométricas de instrumentos.

\begin{tabular}{|l|c|c|c|c|}
\hline \multicolumn{1}{|c|}{ Nombre } & Abreviatura & Validez & Confiabilidad & $\begin{array}{c}\text { Número } \\
\text { de reactivos }\end{array}$ \\
\hline $\begin{array}{l}\text { Inventario de Ansiedad de Beck } \\
\text { (Robles, Varela, Jurado y Páez, 2001). }\end{array}$ & BAI & De constructo & $\begin{array}{c}\text { Consistencia interna } \\
\alpha=0.83\end{array}$ & 21 \\
\hline $\begin{array}{l}\text { Inventario de Depresión de Beck } \\
\text { (Jurado et al., 1998). }\end{array}$ & BDI & Concurrente & $\begin{array}{c}\text { Consistencia interna } \\
\alpha=0.87\end{array}$ & 21 \\
\hline $\begin{array}{l}\text { Escala de Compasión para Población } \\
\text { Mexicana (López y Moreno, 2019). }\end{array}$ & ECOM & De constructo & $\begin{array}{c}\text { Consistencia interna } \\
\alpha=0.90\end{array}$ & 17 \\
\hline $\begin{array}{l}\text { Escala de Felicidad Subjetiva Duradera } \\
\text { y Escala de Felicidad Subjetiva } \\
\text { Fluctuante (Barrera, Díaz, Sánchez-Sosa } \\
\text { y Moreno, 2019). }\end{array}$ & EFSD & DEFSF & $\begin{array}{c}\text { Confiabilidad compuesta } \\
\Omega=0.95\end{array}$ & 16 \\
\hline
\end{tabular}




\section{Procedimiento}

Se solicitó la aprobación del Comité de Ética del Instituto de Asistencia e Integración Social para llevar a cabo la intervención en una unidad especializada en el tratamiento de adicciones perteneciente a este organismo. Con la ayuda de un psicólogo adscrito a dicha unidad se realizó una entrevista inicial de manera individual, con una duración aproximada de 90 minutos, a los pacientes que asistían al servicio de consulta externa, con el propósito de obtener su historia clínica de consumo y un diagnóstico diferencial de trastornos mentales y del comportamiento debidos al consumo de múltiples sustancias (F.19), según la Clasificación Internacional de Enfermedades (CIE-10) (Organización Mundial de la Salud, 2005), y determinado por el psicólogo tratante del servicio de impresión diagnóstica.

Se entrevistó a un total de nueve pacientes, de los cuales cinco cumplían los criterios de inclusión en el estudio, a quienes se explicó la estructura del tratamiento, mismo que consta de nueve sesiones semanales con duración de dos horas cada una. Se les informó sobre la aplicación de una batería de instrumentos y de pruebas de orina para detectar drogas, después de lo cual se obtuvo su consentimiento informado. Del análisis estadístico se excluyó a un participante que no asistió a las nueve sesiones de tratamiento, de modo que en este reporte solo se presentan los resultados de cuatro participantes.

Una semana antes de la primera sesión terapéutica, se citó a los participantes para una entrevista individual, llevada a cabo con la ayuda de una pasante de psicología, ciega al estudio, con el fin de recolectar los datos basales mediante los instrumentos previamente descritos y la prueba antidoping. De igual manera, al concluir la intervención de nueve sesiones, se hizo una evaluación post (B), y una más, de seguimiento, un mes después (C), mediante la aplicación de los inventarios y la prueba antidoping ya mencionados.

\section{Procedimiento clínico}

La intervención se llevó a cabo de manera grupal en sesiones semanales, las cuales fueron instrumentadas por un psicólogo con entrenamiento en terapia de atención plena y compasión. El programa de intervención consistió en nueve sesiones, con duración de dos horas (Tabla 4). Cada sesión se administró en un espacio bien iluminado con asientos cómodos para el terapeuta y los pacientes. También se contó con una computadora y con dispositivos audiovisuales para las presentaciones PowerPoint durante las sesiones de tratamiento.

Tabla 4. Programa de intervención en salud de atención plena y compasión.

\begin{tabular}{|c|l|l|}
\hline Sesión & \multicolumn{1}{|c|}{ Nombre } & \multicolumn{1}{c|}{ Objetivos } \\
\hline 1 & $\begin{array}{l}\text { Psicoeducación en el } \\
\text { consumo de sustancias } \\
\text { psicoactivas. }\end{array}$ & $\begin{array}{l}\text { Que el participante conozca el contenido del programa, además de proporcionarle } \\
\text { información acerca de las drogas psicoactivas, los factores desencadenantes, sus efectos } \\
\text { a corto y a largo plazo, las consecuencias de su uso agudo y crónico y la importancia } \\
\text { de la adhesión al tratamiento. }\end{array}$ \\
\hline 2 & $\begin{array}{l}\text { Psicoeducación } \\
\text { de atención plena } \\
\text { y compasión. }\end{array}$ & $\begin{array}{l}\text { Que el participante conozca los conceptos de atención plena y compasión y su importancia } \\
\text { para contrarrestar la desregulación emocional (Nater, Skoluda y Strahler, 2013). }\end{array}$ \\
\hline 3 & $\begin{array}{l}\text { Filosofía para cesar } \\
\text { el sufrimiento. }\end{array}$ & $\begin{array}{l}\text { Que el participante conozca una forma de alivio al sufrimiento mediante el reconocimiento } \\
\text { yel entendimiento de su origen y metodología para su cesación (Filosofía } \\
\text { de la atención plena). }\end{array}$ \\
\hline 5 & $\begin{array}{l}\text { Conceptos básicos } \\
\text { de atención plena } \\
\text { compasiva. }\end{array}$ & $\begin{array}{l}\text { Que el participante conozca y lleve a la práctica los conceptos básicos de la atención } \\
\text { plena compasiva. }\end{array}$ \\
\hline 5 & $\begin{array}{l}\text { Práctica de la atención } \\
\text { plena y la compasión } \\
\text { en la vida cotidiana. }\end{array}$ & $\begin{array}{l}\text { Que el participante identifique cómo practicar la atención plena compasiva a lo largo } \\
\text { del día. }\end{array}$ \\
\hline 6 & $\begin{array}{l}\text { Atención plena } \\
\text { y compasión en hábitos } \\
\text { saludables. }\end{array}$ & $\begin{array}{l}\text { Que el participante utilice la atención plena y la compasión para atender sus actividades } \\
\text { del diario vivir, como el ejercicio o la alimentación; además, que reconozca el sufrimiento } \\
\text { propio y ajeno para generar acciones, palabras y pensamientos que contribuyan a cambiar } \\
\text { tales experiencias. }\end{array}$ \\
\hline
\end{tabular}




\begin{tabular}{|c|l|l|}
\hline 7 & $\begin{array}{l}\text { Acciones que favorecen } \\
\text { el bienestar. }\end{array}$ & $\begin{array}{l}\text { Que el participante conozca distintas acciones encaminadas a reconocer y abandonar } \\
\text { las conductas y hábitos destructivos, y que genere y promueva acciones encaminadas } \\
\text { al bienestar propio y el de los demás seres. }\end{array}$ \\
\hline 8 & $\begin{array}{l}\text { Comunicación atenta } \\
\text { y compasiva. }\end{array}$ & Que el participante aplique la atención plena compasiva a las relaciones interpersonales. \\
\hline 9 & $\begin{array}{l}\text { Mantenimiento } \\
\text { de la práctica: viviendo } \\
\text { con atención plena } \\
\text { y compasión. }\end{array}$ & $\begin{array}{l}\text { Que el participante cuente con los elementos necesarios para mantener la práctica } \\
\text { de la atención plena, aunada a algunos hábitos saludables, como el ejercicio físico } \\
\text { o una alimentación saludable. }\end{array}$ \\
\hline
\end{tabular}

\section{Análisis estadístico}

Se calculó el tamaño del efecto clínico de Cardiel (Parker y Vannest, 2009) para evaluar el cambio en sintomatología depresiva y ansiosa, patrón de consumo, felicidad fluctuante y felicidad duradera y compasión. Este cálculo se considera como el mejor análisis disponible para los diseños de caso único. A los cambios superiores a $0.20 /-0.20$ se les supone como clínicamente significativos $(\mathrm{CCO}=$ Posest- Pretest / Pretest $)$.

Se obtuvo el promedio de respuesta en los inventarios de sintomatología ansiosa y depresiva, felicidad fluctuante, felicidad duradera y compasión de cada paciente a lo largo de las tres mediciones durante la maniobra terapéutica.

Además, se presenta el patrón de consumo de marihuana, cocaína y alcohol a lo largo de la intervención.

\section{RESULTADOS}

En la Tabla 5 se muestra el cambio clínico entre las mediciones pretest, postest y seguimiento a un mes. Se encontraron cambios significativos posteriores al tratamiento en la reducción de sintomatología depresiva y ansiosa y felicidad fluctuante en los cuatro pacientes. La felicidad duradera tuvo un incremento clínicamente significativo en tres de ellos, en tanto que la compasión solo se incrementó significativamente en un paciente. Durante el seguimiento se observó una disminución de la sintomatología ansiosa y depresiva y de la felicidad fluctuante en tres de los cuatro pacientes. La felicidad duradera y la compasión no mostraron cambios significativos en la evaluación del seguimiento, aunque se mantuvieron de los cambios producidos por la intervención.

Tabla 5. Cambio clínico en las variables psicométricas.

\begin{tabular}{|c|c|c|c|c|c|c|c|c|c|c|}
\hline \multirow{2}{*}{ Participante } & \multicolumn{2}{|c|}{ Depresión } & \multicolumn{2}{|c|}{ Ansiedad } & \multicolumn{2}{c|}{$\begin{array}{c}\text { Felicidad } \\
\text { fluctuante }\end{array}$} & \multicolumn{2}{c|}{$\begin{array}{c}\text { Felicidad } \\
\text { duradera }\end{array}$} & \multicolumn{2}{c|}{ Compasión } \\
\cline { 2 - 13 } & Post & Seg. & Post & Seg. & Post & Seg. & Post & Seg. & Post & Seg. \\
\hline 1 & -.53 & -.50 & -.93 & 3.00 & -.52 & .08 & .64 & .07 & -.12 & -.05 \\
\hline 2 & -.60 & -.75 & -.13 & -1.00 & -.51 & -.24 & .34 & .02 & .32 & .05 \\
\hline 3 & -.64 & -1.00 & -1.00 & .00 & -.35 & -.53 & .08 & .06 & .08 & .03 \\
\hline 4 & -1.00 & .00 & -.97 & -1.00 & -.49 & -.64 & .33 & .00 & .09 & .01 \\
\hline
\end{tabular}

El consumo de marihuana previo al tratamiento se reportó mediante una línea base retrospectiva en dos de los cuatro usuarios (participantes 1 y 3); dicho patrón de consumo cesó durante el tratamiento para el participante 3, manteniéndose el cambio hasta un mes después del tratamiento. El participante 1 registró recaídas durante el tratamiento y en la fase de seguimiento, aunque sin recuperar el patrón de consumo reportado previo al tratamiento (Gráfica 1).
Los cuatro participantes reportaron consumo de cocaína y su disminución al finalizar el tratamiento; cabe señalar que durante este y en la fase de seguimiento los participantes 3 y 4 mantuvieron la abstinencia del consumo, el participante 1 tuvo recaídas esporádicas durante y después del tratamiento, mientras que el participante 3 sufrió una recaída en el tratamiento para el posterior logro de la abstinencia (Gráfica 2). 
Gráfica 1. Patrón de consumo de marihuana (THC).

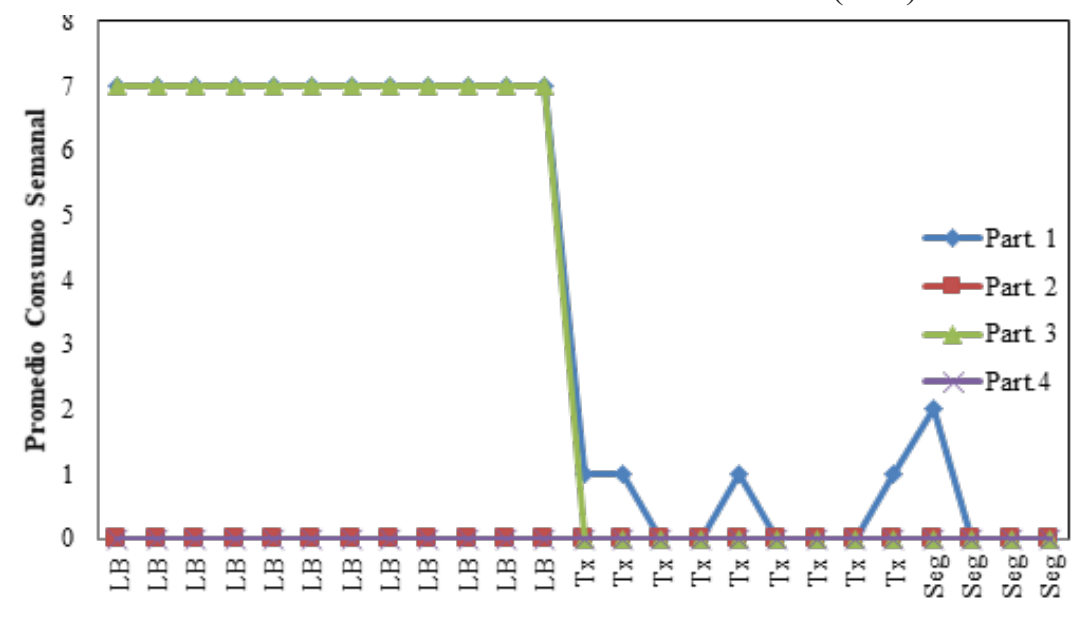

LB = Línea base; $\mathrm{Tx}=$ Tratamiento; $\mathrm{Seg}=$ Seguimiento.

Gráfica 2. Patrón de consumo de cocaína (CO).

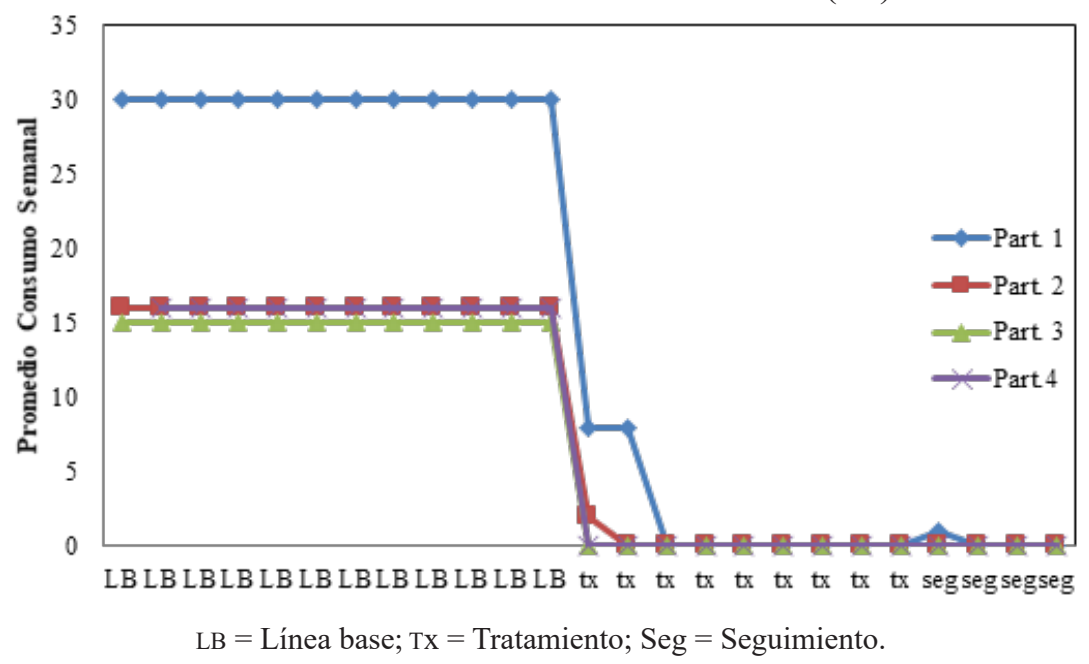

El consumo de alcohol disminuyó en los cuatro participantes a lo largo del tratamiento, y se mantuvo así durante el seguimiento. Los participantes
3 y 4 reportaron abstinencia en ambas fases. Los pacientes 1 y 2 tuvieron recaídas durante y después del tratamiento (Gráfica 3).

Gráfica 3. Patrón de consumo de alcohol (AL).

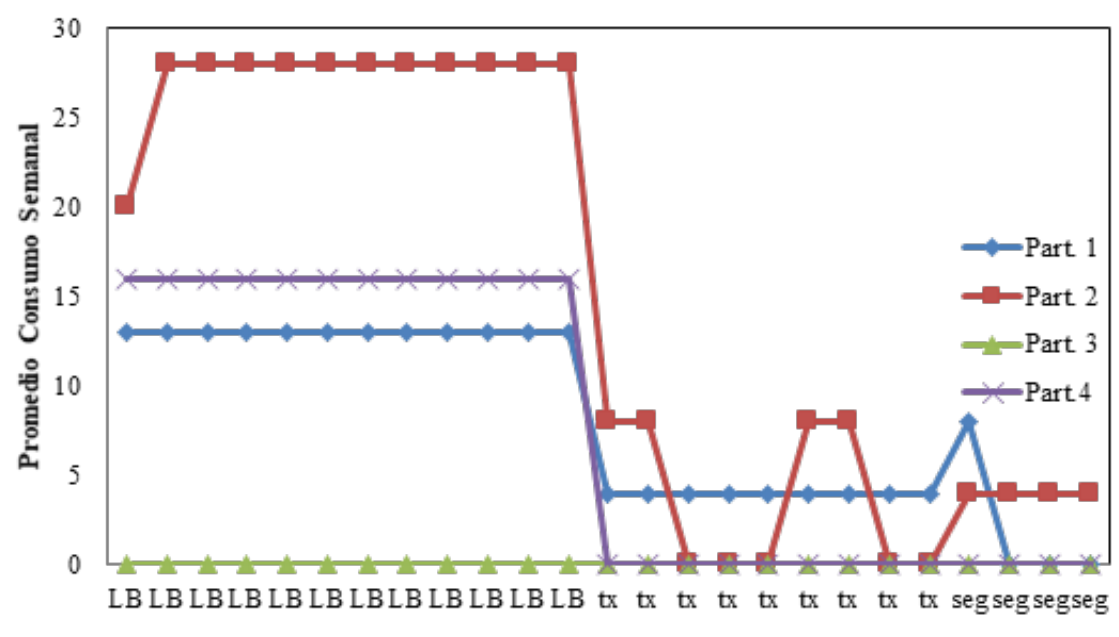

$\mathrm{LB}=$ Línea base $; \mathrm{Tx}=$ Tratamiento Seg $=$ Seguimiento. 


\section{DISCUSIÓN}

En México, el consumo de drogas es un problema de salud pública que repercute en prácticamente todas las áreas de vida de la persona debido al deterioro físico y mental que ocasiona, por lo que es fundamental desarrollar programas de tratamiento que sean útiles para este tipo de usuarios.

Los tratamientos de atención plena para el consumo de sustancias han resultado muy eficaces en todo el mundo, aunque en México no hay estudios que aporten evidencias sobre su efectividad como tratamiento contra el consumo de sustancias, por lo que el presente es un estudio pionero.

Los hallazgos muestran un cambio clínico positivo en la disminución del consumo de sustancias, lo que señala que el modelo basado en la atención plena compasiva es una aproximación valiosa como tratamiento para policonsumidores. De acuerdo con los reportes y las pruebas antidoping utilizadas las personas que participaron en el modelo de atención plena compasiva disminuyeron el consumo de manera clínicamente significativa durante el tratamiento y en el seguimiento.

En la comparación de la línea base retrospectiva y el autorregistro durante el tratamiento y el seguimiento se encontraron diferencias en la disminución de la frecuencia de consumo, lo que trajo consigo que en el caso de los usuarios que tuvieran recaída, tal reincidencia fuera esporádica, y que además consumieran en una cantidad menor a su patrón usual de consumo previo al tratamiento.

Los resultados del presente estudio coinciden con diversas investigaciones hechas en otros países (Enkema y Bowen; 2017; Karyadi, VanderVeen y Cyders 2014; Schellhas, Ostafin, Palfai y Jong, 2016), en las que se sugiere una relación negativa entre el tratamiento en atención plena y las conductas de uso de sustancias, lo que subraya la eficacia del modelo de intervención en personas con un consumo problemático.

Dichos resultados están relacionados con la adquisición y puesta en práctica de los diversos componentes de la atención plena, tales como el mantenimiento y regulación de la atención, la conciencia no elaborativa y la postura de aceptación, así como el desarrollo del conocimiento básico de los principios budistas (Bishop et al., 2004).
Otro indicador de la eficacia del modelo de atención plena compasiva en la presente investigación fue la disminución clínicamente significativa de la sintomatología ansiosa y depresiva una vez finalizado el tratamiento, la que mantuvo dicha disminución durante el seguimiento, coincidiendo así con los resultados de otras investigaciones en las que se muestran cambios significativos en el tratamiento de la ansiedad y depresión (Chiesa y Serreti, 2011; Matvienko-Sikar, Lee, Murphy y Murphy, 2016; Piet y Hougaard, 2011; Simkin y Black, 2014; Williams y Kuyken, 2012).

Los resultados obtenidos apoyan la literatura previa, que señala la relación entre la presencia de problemas emocionales y afectivos y el inicio y mantenimiento del consumo de drogas, donde la autorregulación emocional funge como un factor de protección ante el consumo de sustancias, ayudando así a disminuir la probabilidad de recaídas (Baker, Brandon y Chassin, 2004; Correa et al., 2012; Stotts y Northrup, 2015).

Por último, en las variables de compasión y felicidad se observó un aumento de la compasión y la felicidad duradera, y una disminución de los niveles de felicidad fluctuante después de la intervención, efectos que se mantuvieron en la fase de seguimiento. Lo anterior sugiere, en primer término, que el tratamiento empleado es eficaz para dotar al paciente de los mecanismos compasivos de identificar el malestar propio y el del otro, y la motivación de ejecutar acciones encaminadas a ayudar por medio de conductas prosociales para así promover el bienestar (López y Moreno, 2019); en segundo lugar, muestra la reducción de una felicidad basada en circunstancias externas y el aumento de una que emerge a partir de elementos intrínsecos, que fungen como factores protectores del consumo de sustancias (Dambrum y Ricard, 2011; Enkema y Bowen, 2017).

En conclusión, la atención plena compasiva resultó ser eficaz para reducir el consumo de sustancias, la sintomatología ansiosa y depresiva y la felicidad fluctuante, así como para acrecentar la compasión y la felicidad duradera, por lo que se sugiere incorporar en las mediciones basales y durante el tratamiento el monitoreo de frecuencia cardiaca y la respuesta galvánica, como correlatos biológicos que apoyen las técnicas entrenadas 
durante el tratamiento, toda vez que son datos objetivos que apoyan las mediciones de autorreporte. Se sugiere también aumentar el número de participantes, disponer de un grupo control de intervención con entrevista motivacional y técnicas cognitivo-conductuales de segunda generación, las cuales han mostrado ampliamente su eficacia en el tratamiento de adicciones; efectuar análisis estadísticos más robustos que logren integrar y controlar el efecto de posibles covariables; evaluar la eficacia de la intervención en pacientes con comorbilidad psiquiátrica, ya que en $70 \%$ de los trastornos por consumo de sustancias se presenta, y por último ampliar el seguimiento a tres y seis meses.

\section{REFERENCIAS}

Allen, J., Litten, R. y Fertig, J. (1995). NIDA-NIAAA Workshop: Efficacy of therapies in drug and alcohol addiction. Psychopharmacology Bulletin, 301, 655-669.

Ayala V., H., Cárdenas, G., Echeverría, L. y Gutiérrez, M. (1998). Manual de autoayuda para personas con problemas en su forma de beber. México: Porrúa/UNAM.

Ayala V., H., Echeverría, L., Sobell, M. y Sobell, L. (1988). Una alternativa de intervención breve y temprana para bebedores problema en México. Acta Comportamentalia, 6(1), 71-93.

Baker, T.B., Brandon, T.H. y Chassin, L. (2004). Motivational influences on cigarette smoking. Annual Review of Psychology, 55, 463- 491.

Barragán, L., Flores, M., Morales, D., González, J. y Martínez, M. (2006). Programa de satisfactores cotidianos para usuarios con dependencia a sustancias adictivas: Manual del terapeuta (tomos I y II). México: CONADIC.

Barragán T., L., González J., V., Medina-Mora, M.E. y Ayala V., H. (2005). Adaptación de un modelo de intervención cognoscitivo-conductual para usuarios dependientes de alcohol y otras drogas a población mexicana: un estudio piloto. Salud Mental, $28(1), 61-71$

Barrera G., J.L., Díaz A., D., Sánchez-Sosa, J.J. y Moreno C., A.B. (2019). Adaptación y validación de la estructura factorial de las Escalas de Felicidad Subjetiva Fluctuante (SFHS) y Felicidad Subjetiva Duradera (SA-DHS). Psicología y Salud, 29(2), $195-205$.

Beck, A., Epstein, N., Brown, G. y Steer, R. (1988). An inventory for measuring clinical anxiety: Psychometric properties. Journal of Consulting and Clinical Psychology, 56, 893-897.

Beck, A., Steer, R. y Garbin, M. (1988). Psychometric properties of the Beck Depression Inventory: Twenty-five years of evaluation. Clinical Psychology Review, 8, 77-100.

Bishop, S.R., Lau, M., Shapiro, S., Carlson, L., Anderson, N.D., Carmody, J., Segal, Z.V., Abbey, S., Speca, M., Velting, D. y Devins, G. (2004). Mindfulness: A proposed operational definition. Clinical Psychology: Science and Practice, 11(3), 230-241. Doi: 10.1093/clipsy.bph077.

Bowen, S., De Boer, D. y Bergman, A.L. (2017). The role of mindfulness as approach-based coping in the PTSD-substance abuse cycle. Addictive Behaviors, 64, 212-216.

Brewer, J.A., Elwafi, H.M. y Davis, J.H. (2013). Craving to quit: Psychological models and neurobiological mechanisms of mindfulness training as treatment for addictions. Psychology of Addictive Behaviors, 27(2), 366-379.

Carroll, K.M. y Kiluk, B.D. (2017). Cognitive behavioral interventions for alcohol and drug use disorders: Through the stage model and back again. Psychology of Addictive Behaviors, 31(8), 847-861. Doi: 10.1037/adb0000311.

Chiesa, A. y Serreti, A. (2011). Mindfulness-based cognitive therapy for psychiatric disorders: a systematic review and meta-analysis. Psychiatry Research, 187(3), 441-453.

Chiesa, A. y Serretti, A. (2014). Are mindfulness-based interventions effective for substance use disorders? A systematic review of the evidence. Substance Use \& Misuse, 49, 492-512. Doi: 10.3109/10826084.2013.770027.

Consejo Nacional contra las Adicciones (CONADIC) (2009). Norma Oficial Mexicana NOM-028-SSA2-2009- para la prevención, tratamiento y control de las adicciones. México: CONADIC. Recuperado de http://www.conadic.salud.gob.mx/pdfs/norma_oficial_nom.pdf.

Correa F., V., Castro, Y., Heppner, W.L., Vidrine, J.I. y Costello, T.J. (2012). Mediators of the association of major depressive syndrome and anxiety syndrome with postpartum smoking relapse. Journal of Consulting and Clinical Psychology, 80, 636-648.

Dambrun, M. y Ricard, M. (2011). Self-centeredness and selflessness: a theory of self-based psychological functioning and its consequences for happiness. Review of General Psychology, 15, 138-157.

De las Fuentes, V.M. y Villalpando, J. (2001). Adaptación de un instrumento de tamizaje para la población mexicana que consume drogas. Tesis de Licenciatura. México: UNAM. 
Echeverría, L., Ruiz, G., Salazar, M. y Tiburcio, M. (2008). Manual de detección temprana e intervención breve para bebedores problema. México: CONADIC.

Enkema, M.C. y Bowen, S. (2017). Mindfulness practice moderates the relationship between craving and substance use in a clinical sample. Drug and Alcohol Dependence, 179, 1-7.

Ferrando, L., Bobes, J., Gibert, J., Soto, M. y Soto, O. (2000). MINI. Entrevista Neuropsiquiátrica Internacional (versión en español). Madrid: Sheehan \& Lecrubier.

Gross, J.J. y Levenson, R.W. (1993). Emotional suppression: Physiology, self-report, and expressive behavior. Journal of Personality and Social Psychology, 64(6), 970-986.

Gross, J.J. y Levenson, R.W. (1997). Hiding feelings: The acute effects of inhibiting negative and positive emotion. Journal of Abnormal Psychology, 106(1), 95-103.

Gutiérrez, O., Luciano, M.C., Rodríguez, M. y Fink, B. (2004). Comparison between an acceptance-based and a cognitive-control-based protocol for doping with pain. Behavior Therapy, 35, 767-783.

Hayes, S.C. (2004). Acceptance and commitment therapy, relational frame theory, and the third wave of behavioral and cognitive therapies. Behavior Therapy, 35, 639-665.

Jurado, S., Villegas, M.E., Méndez, L., Rodríguez, F., Loperena, V. y Varela, R. (1998). La estandarización del Inventario de Depresión de Beck para los residentes de la Ciudad de México. Salud Mental, 21, 26-31.

Karyadi, K.A., VanderVeen, J.D. y Cyders, M.A. (2014). A meta-analysis of the relationship between trait mindfulness and substance use behaviors. Drug and Alcohol Dependence, 143, 1-10.

López T., A. y Moreno C., A.B. (2019). Escala de Compasión (ECOM) para población mexicana. Psicología y Salud, 29(1), 25-32.

Magill, M., Ray, L., Kiluk, B., Hoadley, A., Bernstein, M., Tonigan, J.S. y Carroll, K. (2019). A meta-analysis of cognitive-behavioral therapy for alcohol or other drugs use disorders: Treatment efficacy by contrast condition. Journal of Consulting and Clinical Psychology, 87(12), 1093-1105. Doi: 10.1037/ccp0000447.

Marlatt, G.A. y Kristeller, J.L. (1999). Mindfulness and meditation. En W. R. Miller (Ed.): Integrating spirituality into treatment: Resources for practitioners (pp. 67-84). Washington, D.C.: American Psychological Association.

Martínez, K., Salazar, M., Ruiz, G., Barrientos, V. y Ayala, H. (2007). Programa de intervención breve para adolescentes que inician el consumo de alcohol y otras drogas. Manual del terapeuta. México: UNAM.

Matvienko-Sikar, K., Lee, L., Murphy, G. y Murphy, L. (2016). The effects of mindfulness interventions on prenatal well-being: A systematic review. Psychology \& Health, 31(12), 1415-1434.

Moreno, A. (2012). Terapias cognitivo-conductuales de tercera generación (TTG): la atención plena/mindfulness. Revista Internacional de Psicología, 12(1), 1-18.

Oikonomou, M.T., Arvanitis, M. y Sokolove, R.L. (2016). Mindfulness training for smoking cessation: a meta-analysis of randomized controlled trials. Journal of Health Psychology, 22(14), 1841-1850. Doi: 10.1177/1359105316637667.

Organización Mundial de la Salud (2005). CIE-10. Décima revisión de la Clasificación Internacional de las Enfermedades. Trastornos mentales y del comportamiento. Descripciones clínicas y pautas para el diagnóstico. Madrid: Meditor.

Oropeza R., T., Medina-Mora, M.E. y Sánchez-Sosa, J.J. (2007). Evaluación de un tratamiento breve para usuarios de cocaína. Revista Mexicana de Psicología, 24(2), 219-231.

Parker, R.I. y Vannest, K.J. (2009). An improved effect size for single case research: Non-overlap of all pairs (NAP). Behavior Therapy, 40(4), 357-367.

Pascual L., J., Frías N., M.D. y Monterde H., B. (2004). Tratamientos psicológicos con apoyo empírico y práctica clínica basada en la evidencia. Papeles del Psicólogo, 25(87), 1-8.

Pérez A., M. (2006). La terapia de conducta de tercera generación. Revista de Psicología y Psicopedagogía, 5(2), 159-172.

Piet, J. y Hougaard, E. (2011). The effect of mindfulness-based cognitive therapy for prevention of relapse in recurrent major depressive disorder: a systematic review and meta-analysis. Clinical Psychology Review, 31(6), 1032-1040.

Pirnial, B., Akhondi, M., Pirnial, K., Malekanmehr, P., Farzaneh, S., Deilaml, K. y Zahiroddin, A. (2019). A single-case experimental design to study the combination of cognitive-behavioral therapy and pharmacotherapy for smoking cessation. Crescent Journal of Medical and Biological Sciences, 6(1), 136-139.

Robles, R., Varela, R., Jurado, S. y Páez, F. (2001). Versión mexicana del Inventario de Ansiedad de Beck: propiedades psicométricas. Revista Mexicana de Psicología, 18(2), 211-218.

Rojas, E., Real, T., García S., S. y Medina-Mora, M.E. (2011). Revisión sistemática sobre el tratamiento de adicciones en México. Salud Mental, 34(4), 351-365.

Schellhas, L., Ostafin, B.D., Palfai, T.P. y de Jong, P.J. (2016). How to think about your drink: Action-identification and the relation between mindfulness and dyscontrolled drinking. Addictive Behaviors, 56, 51-56.

Schuman-Olivier, Z., Hoeppner, B.B., Evins, A.E. y Brewer, J.A. (2014). Finding the right match: mindfulness training may potentiate the therapeutic effect of nonjudgment of inner experience on smoking cessation. Substance Use \& Misuse, 49(5), 586-594. 
Secretaría de Salud, Instituto Nacional de Psiquiatría Ramón de la Fuente Muñiz, Instituto Nacional de Salud Pública y Comisión Nacional contra las Adicciones (2017). Encuesta Nacional de Consumo de Drogas, Alcohol y Tabaco. Ciudad de México: Autores.

Simkin, D. y Black, N. (2014). Meditation and mindfulness in clinical practice. Child \& Adolescent Psychiatric Clinics of North America, 23(3), 487-534.

Stotts, A.L. y Northrup, T.F. (2015). The promise of third-wave behavioral therapies in the treatment of substance use disorders. Current Opinion in Psychology, 2, 75-81. Doi: 10.1016/j.copsyc.2014.12.028.

United Nations Office for Drugs and Crime (2020). World Drug Report 2020. New York: UNODC.

Williams, M. y Kuyken, W. (2012). Mindfulness-based cognitive therapy: a promising new approach to preventing depressive relapse. The British Journal of Psychiatry, 200, 359-360.

Witkiewitz, K., Marlatt, G.A. y Walker, D.D. (2005). Mindfulness-based relapse prevention for alcohol and substance use disorders. Journal of Cognitive Psychotherapy, 19, 211-228.

Zgierska, A., Rabago, D., Chawla, N., Kushner, K., Koehler, R. y Marlatt, A. (2009). Mindfulness meditation for substance use disorders: a systematic review. Substance Abuse, 30(4), 266-294. 